\title{
Wang and Mendel's Fuzzy Rule Learning Method for Energy Consumption Forecasting considering the Influence of Environmental Temperature
}

\author{
IPP - Polytechnic of Porto, \\ Porto, Portugal \\ \{arjoz, tmcfp, icp, fspsa, bccta, zav\}@isep.ipp.pt
}

Aria Jozi, Tiago Pinto, Isabel Praça, Francisco Silva, Brigida Teixeira, Zita Vale

GECAD - Research Group on Intelligent Engineering and Computing for Advanced Innovation and Development,

\begin{abstract}
Reliable consumption forecasts are crucial in several aspects of power and energy systems, e.g. to take advantage of the full potential of flexibility from consumers and to support the management from operators. With this need, several methodologies for electricity forecasting have emerged. However, the study of correlated external variables, such as temperature or luminosity, is still far from adequate. This paper presents the application of the Wang and Mendel's Fuzzy Rule Learning Method (WM) to forecast electricity consumption. The proposed approach includes two distinct strategies, the first one uses only the electricity consumption as the input of the method, and the second strategy considers a combination of the electricity consumption and the environmental temperature as the input, in order to extract value from the correlation between the two variables. A case study that considers the forecast of the energy consumption of a real office building is also presented. Results show that the WM method using the combination of energy consumption data and environmental temperature is able to provide more reliable forecasts for the energy consumption than several other methods experimented before, namely based on artificial neural networks and support vector machines. Additionally, the WM approach that considers the combination of input values achieves better results than the strategy that considers only the consumption history, hence concluding that $\mathrm{WM}$ is appropriate to incorporate different information sources.
\end{abstract}

Index Terms- Electricity Consumption, Environmental Temperature, Forecasting, Fuzzy Rule Based Systems, Wang and Mendel's Fuzzy Rule.

\section{INTRODUCTION}

The large scale integration of renewable energy sources has led to an increase of the unpredictability of energy generation [1]. This increase of renewable generation is mostly due to environmental concerns about the impact of greenhouse gas emissions, and the scarcity of fossil fuels. This has led the European Union (EU) to assumed a pioneer and leading role in

The present work has been developed under the EUREKA - ITEA2 Project FUSE-IT (ITEA-13023), Project GREEDI (ANI|P2020 17822), and has received funding from FEDER Funds through COMPETE program and from National Funds through FCT under the project UID/EEA/00760/2013. this field and commit to reach ambitious targets [2]. This uncertainty in the generation side forces a shift in the power system paradigm, to make the consumption become as adaptive as possible, in order to compensate for the continuous fluctuation of generation. Thereby, the demand response concept emerges [3].

In order to take advantage of the full potential of flexibility from consumers, effective consumption forecasting methodologies are crucial. Reliable consumption forecasts are also essential to enable electricity operators to have a better control on the power distribution network [4], e.g. by supporting the management of reserved electricity to be used in emergency scenarios. In this way, the generation cost for electricity can be minimized, and dynamic electricity tariffs that bring the most advantages out of consumers' participation can be defined [5]. Energy consumption forecasts can also reduce the energy waste, control the energy reserve and master the energy consumption trend [6].

Many studies related to energy consumption forecasting have been published. In [7] the author advances the state-ofthe-art by correcting biases in the weather forecasts and interpolating the daily weather forecasts into higher frequency to synchronize with the frequency of the energy consumption forecasts. In [8] an evolutionary neural network is developed as a structurally optimal black-box model to forecast energy consumption of a dynamic cloud data center. This neural network includes several novel mechanisms of a genetic algorithm, such as a structurally inclusive matrix encoding and species parallelism. Additionally, in [9] an artificial neural network based methodology has been implemented to forecast the consumption in an office building. Most of these works achieve acceptable results. However, the integration of correlated external variables, which can aid the forecast of energy consumption, such as the influence of temperature, brightness, among others, is still insufficiently studied. 
Fuzzy Rule Based Systems (FRBS) are methods classified within Computational Intelligence, based on fuzzy concepts, and have been proposed in [10] to address complex real-world problems containing uncertainty, imprecision, and nonlinearity. They aim at representing knowledge in a set of fuzzy rules [11]. In 1992, Li-Xin Wang and Jerry M. Mendel proposed a method for generating fuzzy rules from examples [12]. This method, known as Wang and Mendel's Fuzzy Rule Learning Method (WM) is able to combine numerical and linguistic information, culminating in a set of rules to be used in a FRBS [13].

In this paper, the WM method proposed by Wang and Mendel has been implemented to forecast the energy consumption of an office building based on two different strategies, to enable an assessment of the capability of this method in incorporating different sources of information in the training process. The first proposed strategy uses only the historic of energy consumption as the input of the method. On the other hand, the second strategy uses as input of the method, a combination of energy consumption and the environmental temperature of the related place. The second strategy thus uses a combination of a variable that has a direct influence on electricity consumption.

After this introductory section, section II presents the two proposed strategies, including the description of the used WM learning method used as basis for the forecast process. Section II also includes a description of the data that is used in the experimental tests, as presented in section III. Finally, section IV presents the most relevant conclusions and contributions of this work.

\section{MATERIAL AND METHODS}

This paper addresses the electricity consumption problem, using the Wang and Mendel's Fuzzy Rule Learning Method (WM). The electricity consumption form building $\mathrm{N}$ of the Research Group on Intelligent Engineering and Computing for Advanced Innovation and Development (GECAD) research center located in ISEP/IPP, Porto, Portugal has been used in this work. The environmental temperature of the same place is also used [14]. The WM approach has been developed based on $\mathrm{R}$ programming language. The electricity consumption forecast is calculated based on two different strategies:

- WM1: this strategy uses only the electricity consumption as the input of the method.

- WM2: this strategy considers a combination of the electricity consumption and the environmental temperature as input of the method.

The implementation details and results of these two strategies are discussed and compared in the following sub-sections.

\section{a) Wang and Mendel's Method}

The WM model has been widely known because of being simple and having a good performance [6]. This method is based on working with an input-output data set, as in Equation.1.

$$
E=\left\{e_{1}, \ldots, e_{p}\right\}, e_{1}=\left\{x_{1}^{l}, \ldots, x_{n}^{l}, y^{l}\right\} \quad \text { (Equation.1) }
$$

The generation of the fuzzy rules bases is putted into effect by means of the following steps [15]:

\section{Divide the Input and Output Spaces into Fuzzy Regions.}

It may be obtained from the expert information (if it is available) or by a normalization process. If the latter is the case, perform a fuzzy partition of the input variable spaces dividing each universe of discourse into a number of equal or unequal partitions, select a kind of membership function and assign one fuzzy set to each subspace. In our case, we will work with symmetrical fuzzy partitions of triangular membership functions (Figure 1).

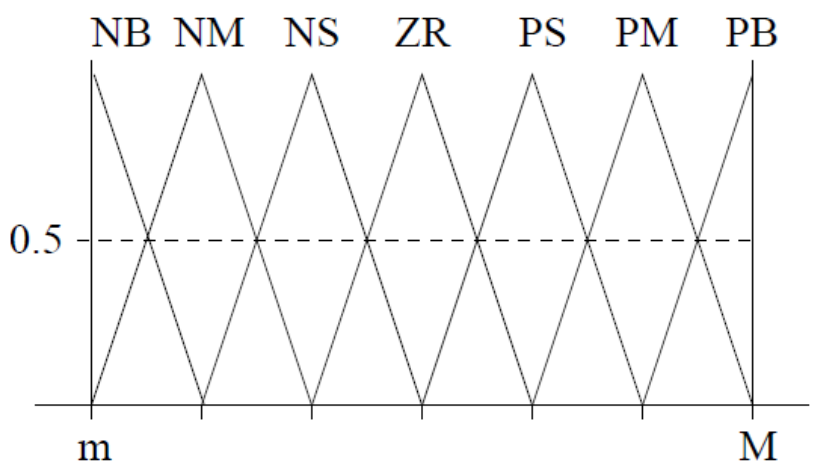

Figure 1: Graphical overview of a uniform fuzzy partition [6]

\section{Generate Fuzzy Rules from Given Data Pairs}

In this step, the generation of fuzzy rules includes the training data, using the database from Step 1. First, the degrees of the membership functions are calculated for all values in the training data. For each instance in the training data, a linguistic term having a maximum degree in each variable will be determined. Moreover, the process will be repeated for every instance in the training data to construct fuzzy rules covering the training data [11].

\section{Assign a degree to each rule}

Determine a degree for each rule. Degrees of each rule are determined by aggregating the degree of membership functions in the antecedent and consequent parts. In this case, we are using the product aggregation operators. If $R_{1}=$ I F $x_{1}$ is $A_{1}$ and ... and $x_{n}$ is $A_{n}$ THEN $y$ is $B$, be the linguistic rule generated from the example $e_{l}, l=1, \ldots, p$. The importance degree associated to it will be obtained as Equation.2: 


$$
G\left(R_{l}\right)=\mu A_{1}\left(x_{1}^{l}\right), \ldots, \mu A_{1}\left(x_{n}^{l}\right) \cdot \mu B\left(y^{l}\right) \quad \text { (Equation.2) }
$$

\section{Create a Combined Fuzzy Rule Base}

The $\rho$ candidate rules are first divided in $g$ different groups, each one of these groups composed of all the candidate rules presenting the same antecedent. It will note by $R_{j i}$, the $j$ th rule in the $i$-th group. To compose the final rules, the rule with the highest importance degree is chosen in each group $i$, $i=1, \ldots, g$. Hence, $g$ will be both the number of different antecedent combinations in the candidate rule set and the number of linguistic rules in the final generated rule base.

Interpolative reasoning that FRBS develops, is one of the interesting characteristics of this method, which plays a key role in the high performance of FRBSs. It is a consequence of the cooperation among the fuzzy rules composing the knowledge Base. The FRBS output is not usually because of a single fuzzy rule, and due to the cooperative action of several fuzzy rules that have been exported, they match the system input to any degree. Regarding to the rule with the best performance in each subspace and, the covering degree, WM Method hooks up the example data set into the fuzzy subspaces (the antecedent combinations mentioned in step 4 of the algorithm). Consequently, the global interaction among the rules of the Knowledge Base is not mentioned. This causes that the obtained rule set, do not cooperate appropriately. Therefore, these rules make the method be more sensitive to noise due to the local processing.

\section{b) Data base description}

The SQL server of GECAD research center located in ISEP/IPP, Porto, Portugal has been used in this study. This server has the databases with all the electricity data of GECAD research center. In this work the data of building $\mathrm{N}$ of this research center is used. This building includes five energy meters and each of them stores the electricity consumption data from one specific part of the building with 10 seconds time interval. Also there is another database in this SQL server that contains the complete weather information obtained from ISEP meteorology website [14]. The environmental temperature of the related place with the same time interval id chosen of this database.

A Java application has been developed in this implementation to collect the data from the databases and calculate the average of environmental temperature as well as the total electricity consumption of the building $\mathrm{N}$ ISEP/GECAD per each hour. This application also creates a new .csv file in a format that can be used as the input of the forecast method.

\section{RESULTS AND DISCUSSION}

The main objective of this study is to forecast the profile of the electricity consumption for the following hour. The WM method has been employed for this forecast based on two deferent strategies to compare the results. In the WM1 strategy only the electricity consumption from the first hour of the day until the hour that is meant to be forecasted, is used as the input of the WM method. In contrast, the WM2 uses as input of the WM method, a combination of the electricity consumption and the environmental temperature of the related place from beginning of the day until the hour prior to the forecast. In WM2 it has been tried to use a combination of a variable that may affect the forecasted variable, in order to study the possibility of improving forecasting results by extracting correlations between the different variables. In this work, the environmental temperature, as one of the variables that influences the energy consumption, is used as forecast input.

\section{a) WM1 strategy}

In the first strategy, the electricity consumption is used as the only input of the method. A Java application, which is connected to de GECAD SQL server database, has been implemented to extract the electricity consumption from each energy meters of GECAD's building $\mathrm{N}$ of ISEP/IPP Porto, Portugal. This application calculates the total energy consumption of the building as well as the average consumption value for each hour.

For each forecasted value, a set of the 10 days prior to the target forecasting date has been used as the input of the WM method. Each time that the method is trained, the input values for the actual forecast consider the consumption values since the beginning of the day until one hour before the hour of the forecast. The same data set is used as the main input of the method. Table 1 shows an excerpt of the used data in the case of forecasting hour 3 of day 20/11/2015.

Table.1 - Excerpt of WM1 test input table for hour 3 of 12/11/2015

\begin{tabular}{|c|c|c|c|}
\hline Hour & 0 & 1 & 2 \\
\hline $\begin{array}{c}\text { Consumption } \\
\text { (W) }\end{array}$ & 1406.77 & 1169.857 & 1640.13 \\
\hline
\end{tabular}

The value of energy consumption of a total of 12 hours has been forecasted, namely from 12:00 until 23:00 of 20/11/2015, in order to test the WM1 approach. These hours have been chosen because they refer to the hours of greatest activity in the building (higher variation in consumption), thus being the most interesting hours to forecast. An excel file has been created to forecast the energy consumption of each hour, which include three tables: (i) Training input, (ii) Training output, and (iii) Testing input. The MAPE (Mean Absolute Percent Error) error calculation formula is used to compare the forecasted values and the real values of each hour.

Table 2 shows a comparison between the forecasted values and the real values for each of the 12 hours, including the MAPE error value for each of these hours. As table 2 demonstrates the average of forecast errors for the WM1 approach is $18.41 \%$, the largest forecasting error refers to hour 
14 , and the smallest error is of $2.31 \%$, in hour 16 of the considered day.

Table 2 - WM1 results for the 12 considered hours of 20/11/2015

\begin{tabular}{|c|c|c|c|}
\hline Hour & $\begin{array}{c}\text { Real } \\
\text { Consumption } \\
(\mathrm{W})\end{array}$ & $\begin{array}{c}\text { WM1 } \\
\text { Forecasted } \\
\text { Consumption } \\
(W)\end{array}$ & $\begin{array}{c}\text { MAPE } \\
\text { Forecasting } \\
\text { Error }(\%)\end{array}$ \\
\hline 12 & 2244.532 & 2566.2907 & $14.34 \%$ \\
\hline 13 & 2137.4679 & 2953.1492 & $38.16 \%$ \\
\hline 14 & 2089.8918 & 3423.8942 & $63.83 \%$ \\
\hline 15 & 3257.5824 & 3147.2431 & $3.39 \%$ \\
\hline 16 & 3441.8819 & 3521.255 & $2.31 \%$ \\
\hline 17 & 3739.1163 & 2915.9124 & $22.02 \%$ \\
\hline 18 & 2134.12 & 2567.2993 & $20.30 \%$ \\
\hline 19 & 2313.9283 & 1879.9583 & $18.75 \%$ \\
\hline 20 & 1291.5552 & 1164.2223 & $9.86 \%$ \\
\hline 21 & 1268.6335 & 1180.2035 & $6.97 \%$ \\
\hline 22 & 1207.0021 & 1342.6765 & $11.24 \%$ \\
\hline 23 & 1258.8538 & 1381.9637 & $9.78 \%$ \\
\hline
\end{tabular}

\section{b) WM2 strategy}

In the WM2 strategy, the input of the WM method is a combination of the energy consumption and a variable that may affect the electricity consumption. In this specific research work, the environmental temperature has been used as the second variable. This strategy uses the same way as the WM1 to select and calculate the energy consumption. Also the environmental temperature is extracted from ISEP meteorology website [14] in a 10 seconds time interval. The average of the energy consumption as well as the average of environmental temperature values for each hour is calculated by the Java application. For this strategy a set of the last 10 days prior to the date that is meant to be forecasted is also used as the train input of the method. The method is trained using a table that includes the values of the energy consumption and the environmental temperature of every hour from the first hour of the day until one hour before the target forecasting date. An excerpt of the used data is shown in Table 3, for the case of forecasting hour 3 of the day 20/11/2015.
Table 3 - - Excerpt of WM2 test input table for hour 3 of 20/11/2015

\begin{tabular}{|c|c|c|c|}
\hline Hour & 0 & 1 & 2 \\
\hline $\begin{array}{c}\text { Temperature } \\
\left({ }^{\circ} \mathbf{C}\right)\end{array}$ & 11.19 & 10.825 & 10.6 \\
\hline $\begin{array}{c}\text { Consumption } \\
(\mathbf{W})\end{array}$ & 1406.779 & 1169.857 & 1640.133 \\
\hline
\end{tabular}

The same hours as for the WM1 strategy have been used for testing purposes, namely 12 hours of 11 November 2015, to test and compare the WM2 strategy. Table 4 shows a comparison between the forecasted values and the real values for each of the 12 hours, including the MAPE error value for each hour.

Table 4 - WM2 results for the 12 considered hours of 20/11/2015

\begin{tabular}{|c|c|c|c|}
\hline Hour & $\begin{array}{c}\text { Real } \\
\text { Consumption } \\
\text { (W) }\end{array}$ & $\begin{array}{c}\text { WN2 } \\
\text { Forecasted } \\
\text { Consumption } \\
\text { (W) }\end{array}$ & $\begin{array}{c}\text { MAPE } \\
\text { Forecasting } \\
\text { Error (\%) }\end{array}$ \\
\hline 12 & 2244.532 & 2332.6174 & $3.92 \%$ \\
\hline 13 & 2137.4679 & 2186.4059 & $2.29 \%$ \\
\hline 14 & 2089.8918 & 2489.1027 & $19.10 \%$ \\
\hline 15 & 3257.5824 & 3368.6922 & $3.41 \%$ \\
\hline 16 & 3441.8819 & 3157.2597 & $8.27 \%$ \\
\hline 17 & 3739.1163 & 3210.2749 & $14.14 \%$ \\
\hline 18 & 2134.12 & 2267.2993 & $6.24 \%$ \\
\hline 19 & 2313.9283 & 1929.492 & $16.61 \%$ \\
\hline 20 & 1291.5552 & 1147.4501 & $11.16 \%$ \\
\hline 21 & 1268.6335 & 1123.7672 & $11.42 \%$ \\
\hline 22 & 1207.0021 & 1240.3139 & $2.76 \%$ \\
\hline 23 & 1258.8538 & 1260.3764 & $0.12 \%$ \\
\hline
\end{tabular}

The average of the MAPE errors achieved by the WM2 is $8.29 \%$, it is visible that the variation of the forecasted values is much smaller than that verified by using the WM1 approach. The largest forecasting error is still verified in hour 14, however, this error is less than one third of the forecasting error verified for the same hour using WM1. The smallest error is, in this case, in hour 23, and assumes an error value of $0.12 \%$.

\section{c) Comparison of results}

By comparing the average MAPE forecasting error of the both strategies at the same hours and same day it is visible that the WM2 has a much smaller forecasting error than the WM1. 
The average MAPE forecasting error for these hours when using the WM1 is $18.41 \%$, while when using the WM2 the average error is $8.29 \%$. It means that, when the environmental temperature is used in the input of the method, the final forecasted value is more reliable. As Figure 2 demonstrates, in most of the hours, the forecasted value by the WM2 is much closer to the real consumption vale, than the value forecasted by the WM1.

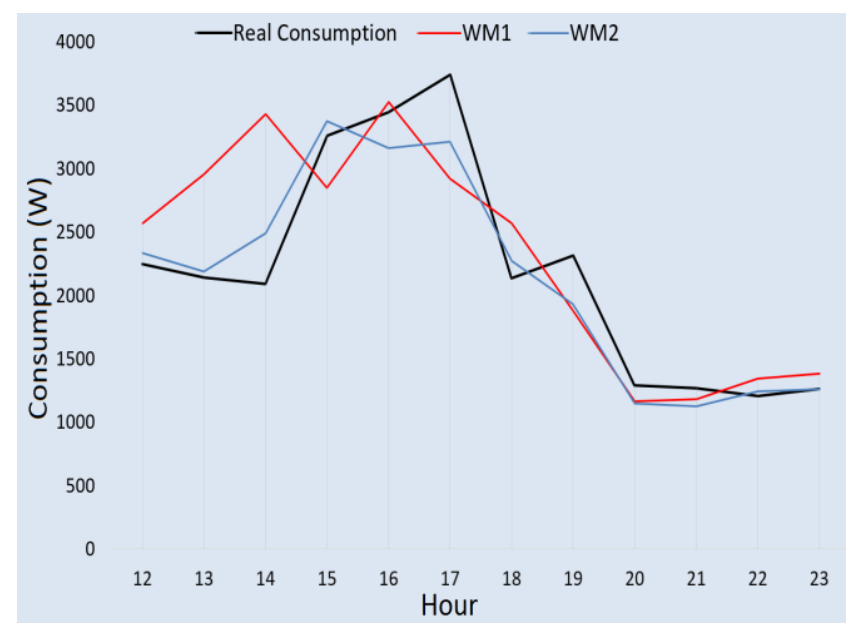

Figure 2 -Comparison of both strategies results.

As Figure 2 shows, using the environmental temperature can have a significant influence on the forecasted value, especially during the hours that there is more activity in the building (during hours 12:00 to 18:00). In these hours, using the variation of the temperature (input value used by WM2), some appliances, for instance the air conditioning system, can be widely used. Thus comparing with other moments, these appliances present a large consumption. The results in WM2 achieving lower forecasting errors during most hours, as shown by Figure 3. As Figure 3 shows, during the hours that the energy consumption is higher, the difference in forecasting error from both strategies is also much more evident than in other moments. The WM2 is able to forecast a more reliable value than WM1 particularly during these hours.

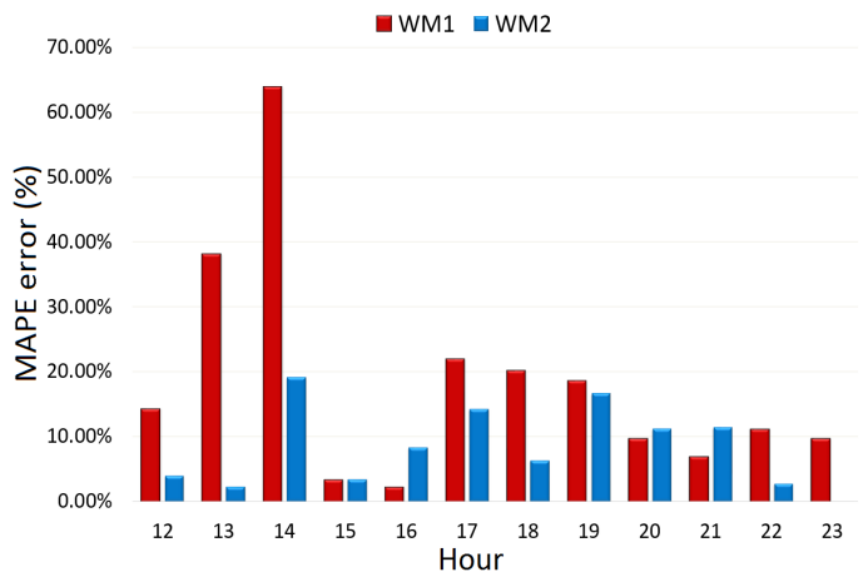

Figure 3 - Forecasting errors of both strategies during the considered hours
In fact, the proposed WM2 approach is also able to achieve better results than other previously experimented forecasting approaches. The study presented in [9] has dealt with the electricity consumption forecast problem using a set of different Artificial Neural Network (ANN) based approaches, which are summarized in Table 5.

Table 5-Different ANN neurons input / output definition, from [9]

\begin{tabular}{|c|c|c|c|c|}
\hline \multirow{2}{*}{$\begin{array}{c}\text { Id } \\
\text { ANN }\end{array}$} & \multicolumn{2}{|c|}{ Input neurons } & \multicolumn{2}{|c|}{ Output neurons } \\
\hline & No. & Description & No. & Description \\
\hline ANN_1 & 3 & $\begin{array}{l}\text { - Context } \\
\text { data* }^{*}\end{array}$ & 1 & $\begin{array}{c}\text { - Total } \\
\text { consumption } \\
\text { per hour }\end{array}$ \\
\hline ANN_2 & 3 & - Context data & 3 & $\begin{array}{l}\text { - Consumption } \\
\text { of HVAC; } \\
\text { - Consumption } \\
\text { of lights; } \\
\text { - Consumption } \\
\text { of sockets }\end{array}$ \\
\hline ANN_h & 4 & $\begin{array}{c}\text { - Context } \\
\text { data; } \\
\text { - External } \\
\text { environmental } \\
\text { data }^{* *}\end{array}$ & 1 & $\begin{array}{c}\text { - Consumption } \\
\text { of HVAC }\end{array}$ \\
\hline ANN_1 & 4 & $\begin{array}{c}\text { - Context } \\
\text { data; } \\
\text { - External } \\
\text { environmental } \\
\text { data } \\
\end{array}$ & 1 & $\begin{array}{c}\text { - Consumption } \\
\text { of light }\end{array}$ \\
\hline ANN_s & 4 & $\begin{array}{c}\text { - Context } \\
\text { data; } \\
\text { - External } \\
\text { environmental } \\
\text { data } \\
\end{array}$ & 1 & $\begin{array}{c}\text { - Consumption } \\
\text { of sockets }\end{array}$ \\
\hline
\end{tabular}

* The context data refer to: hour; day of the week and hour of work (the hour of work is characterized as: time of high, medium and low permanency in the building);

** The external environment data refers to the following variables: temperature, temperature felt, radiation, precipitation and humidity.

Figure 4 shows the electricity consumption forecasting error results achieved by the several ANN approaches (from [9]) and the forecasting results achieved by WM1 and WM2 strategies presented in this paper.

By comparing the result of the several ANN and the results of WM1 and WM2 one can see that the W2 strategy is able to forecast a better energy consumption profile than all the other 
strategies, by achieving smaller forecast errors than all other compared methods.

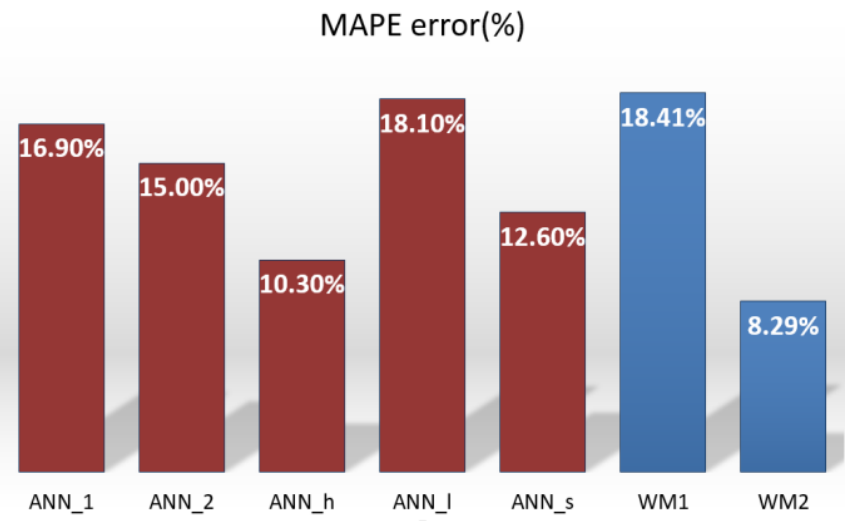

Figure 4 - Average forecasting errors of the ANN and WM methods

\section{CONCLUSION}

This paper focuses on studying the effect of using related variables to support the forecasting of energy consumption. This work has thus proposed two different strategies (WM1 and WM1) to forecast the energy consumption. The first strategy considers only the energy consumption from the lasts hours as the input of the WM method. On the other hand, the second strategy uses a combination of the energy consumption and the environmental temperature of the related place as the input of the WM method.

The achieved results, shown in this paper, illustrate that using the environmental temperature data can help the WM method to have a more reliable result, since the forecasted values achieved by the WM2 strategy are closer to the real values, when compared to the consumption values forecasted by the WM1 strategy. The comparison of these results shows that the quality of results of the proposed approach is especially relevant during times when there is more activities in the building. During these moments, several appliances, namely air condition systems, can be used in order to regulate the temperature of the environment. Therefore, by using the environmental temperature information and finding associations between these data and energy consumption values, the WM method can predict a more reliable value for the energy consumption of the following hours.

As future work, the influence of other external factors, such as brightness and humidity, will also be studied. Associations between several variables simultaneously will also be considered, in order to extract conclusions in their impact on energy consumption forecasting.

\section{REFERENCES}

[1]. Conejo, A., Carrión, M., and Morales, J. 2010. Decision making under uncertainty in electricity markets.

[2]. European Commission, "2030 framework for climate and energy policies", 2014, Available: http://ec.europa.eu/clima/policies/2030/index_en.htm [accessed in March 2016]

[3]. Pedro Faria, Zita Vale, José Baptista, "Demand Response Programs Design and Use Considering Intensive Penetration of Distributed Generation", Energies, vol. 8, no. 6, pp. 6230-6246, June 2015

[4]. S. X. Yang and Y. Wang, "Applying Support Vector Machine Method to Forecast Electricity Consumption," Computational Intelligence and Security, 2006 International Conference on, Guangzhou, 2006, pp. 929-932.

[5]. M. S. M. Othman, D. Johari, I. Musirin, T. K. A. Rahman and N. F. N. Ismail, "Artificial neural network-based forecast for electricity consumption in Malaysia," Power and Energy (PECon), 2010 IEEE International Conference on, Kuala Lumpur, 2010, pp. 24-28.

[6]. Xiao Hong Yang, Shi Xu Guo, Yong Hong Zhang, Hong Tao Yang, A Sort of New Energy Consumption Forecast Method, 2008 Chinese Control and Decision Conference (CCDC 2008).

[7]. Rui Zhang, Hongxia Yang, Dynamic Building Energy Consumption Forecast Using Weather Forecast Interpolations, 2015 IEEE International Conference on Smart Grid Communications (SmartGridComm): Data Management, Grid Analytics, and Dynamic Pricing.

[8]. Yong Wee Foo, Cindy Goh, Hong Chee Lim, Zhi-Hui Zhan, Yun Li, Evolutionary Neural Network Based Energy Consumption Forecast for Cloud Computing, 2015 International Conference on Cloud Computing Research and innovation.

[9]. E. Vinagre, L. Gomes and Z. Vale, "Electrical Energy Consumption Forecast Using External Facility Data," Computational Intelligence, 2015 IEEE Symposium Series on, Cape Town, 2015, pp. 659-664.

[10]. L. A. Zadeh, "Fuzzy Sets," Information and Control, vol. 8, pp. 338353,1965

[11]. Lala Septem Riza, Christoph Bergmeir, Francisco Herrera, and Jose Manuel Benítez, Learning from Data Using the R Package "frbs", 2014 IEEE International Conference on Fuzzy Systems (FUZZ-IEEE) July 6-11, 2014, Beijing, China

[12]. L. X. Wang, J. M. Mendel, "Generating fuzzy rules by learning from examples", IEEE Transactions on Systems, Man and Cybernetics, vol.22, no.6, p.1414-1427, 1992.

[13]. E. R. R. Kato, O. Morandin Jr., M. Sgavioli, B. D. Muniz, Genetic Tuning for Improving Wang and Mendel's Fuzzy Database, Proceedings of the 2009 IEEE International Conference on Systems, Man, and Cybernetics San Antonio, TX, USA - October 2009

[14]. ISEP meteo website: http://meteo.isep.ipp.pt

[15]. J. Casillas, O. Cord'on, F. Herrera, Improving the Wang and Mendel's Fuzzy Rule Learning Method by Inducing Cooperation Among Rules. 